\title{
Better visual aid for cancer surgery
}

\author{
Progress in fluorescence-guided systems and contrast agents for real-time intraoperative assistance during \\ tumour-resection operations should benefit patient outcomes.
}

n $\mathrm{n}$ interventional oncology, making or confirming a diagnosis, surgically resecting cancerous tissue, locating metastatic lesions, and helping plan further treatment may all require surgical interventions. For example, image-guided biopsy followed by histopathological analysis is often used to confirm an initial diagnosis made via computed-tomography imaging. During the surgical resection of tumour tissue, optical imaging can provide immediate feedback guidance to the surgical team. Intraoperative optical guidance can in fact be critical, in particular because it aids the discrimination of tumour tissue from surrounding blood vessels and nerves that need to be preserved, and because the status of the surgical margins (which can range between a few millimetres to a few centimetres) after the surgical resection of cancerous tissue is associated with diseasefree survival rates. The ideal surgical outcome involves 'negative margins', meaning that the tissue surrounding the resected site is likely to be clear of malignant cells and that the entire tumour mass has been excised.

The success of image-guided surgery, and the size of the resection margins, mainly depend on the combined characteristics of the patient's tumours (size, location, accessibility, grade and stage), on the suitability of the optical-imaging probes used to enhance contrast, and on the imaging set-up available to the surgical team. The clearer the demarcation between healthy tissue, diseased tissue and the peritumoral stroma (which may also contain malignant cells), the easier it is to resect the entire tumour. The ability to visually enhance malignant tissue intraoperatively gives the surgeon greater confidence of having obtained negative margins, and decreases the likelihood that the patient needs additional surgical interventions to remove further tumour mass (because of remaining cancerous tissue after confirmation via histopathological analysis, or because of tumour regrowth).

Biocompatible optical-imaging agents with high selectivity and specificity for discriminating cancerous and healthy tissue could thus improve surgical-resection outcomes. Samuel Achilefu and colleagues report in an Article in this issue that a cyclic octapeptide labelled with a near-infrared dye preferentially binds (at high concentration

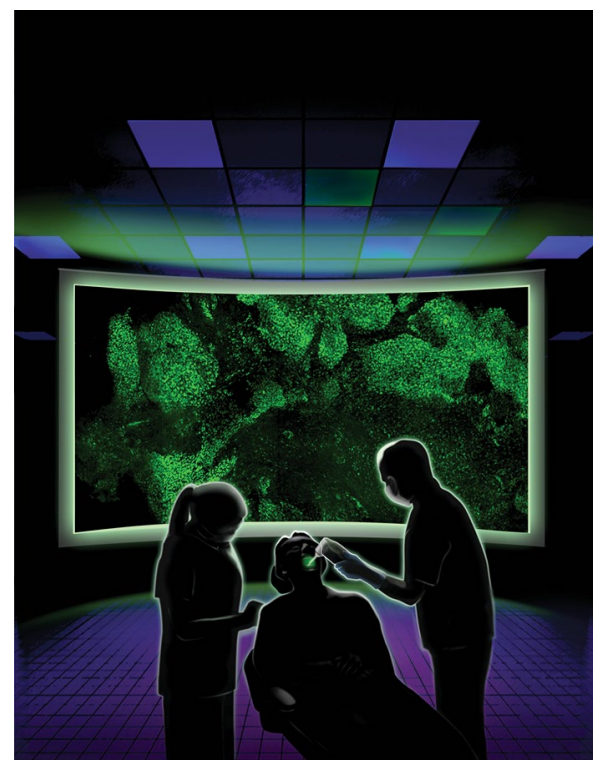

Credit: (c) MSK Design and Creative Services

of calcium) to phosphorylated Annexin A2 (pANXA2), a protein that is upregulated in many cancers. The researchers show, for breast cancer, pancreatic cancer and fibrosarcoma human xenografts in mice, that pANXA2 locates along the proliferating edge of the tumours and in cancerassociated fibroblasts and macrophages, and that its phosphorylation facilitates tumour invasion and metastasis. The use of the pANXA2-binding cyclic octapeptide in mice during fluorescence-guided surgery helped to identify tumour lesions and lymph nodes containing metastatic cells, to resect the tumours, and to inspect the surgical bed for the presence of any residual cancer.

At present, only two near-infrared dyes (indocyanine green and methylene blue) have received market approval by the United States Food and Drug Administration for intraoperative imaging. However, these dyes are not specific for cancer cells, and are not approved for tumour-resection operations. Although fluorescence-guided cancer surgery with non-specific dyes has improved the identification of lesions in patients, it is still unclear whether it improves postoperative outcomes and prognosis (S. Hernot et al. Lancet Oncol. 20, e354-e367; 2019). Fluorescence-guided tumour resection in patients would in fact benefit from dyes that target tumour tissue specifically. In another Article in this issue, Thomas Reiner and colleagues show that a fluorescently labelled clinically used chemotherapy drug (a small-molecule inhibitor that specifically targets the DNArepair enzyme poly(ADP-ribose) polymerase 1 (PARP1), which is highly expressed in tumour tissue) delivered topically or intravenously can be used to detect cancers of the upper intestinal tract (oral, oropharyngeal and oesophageal cancers) in mice and pigs, in biopsied human tissue and in a human. In particular, the fluorescent contrast agent could lead to the identification (within minutes) of positive resection margins in freshly excised tissue. And in data from a patient enrolled in an ongoing openlabel phase-I/II clinical trial (NCT03085147) of the safety and performance of the fluorescently labelled PARP1 inhibitor delivered as a mouthwash (pictured), the targeted imaging agent distinguished benign granuloma from a recurrent malignant oral squamous cell carcinoma.

The surgical resection of primary and metastatic lesions in the liver is typically the preferred treatment option for patients with cancer who have good liver function. However, the surgery is complicated by the liver's strong blood supply and by the size of the organ. As shown by Jie Tian, Zhen Cheng, Sam Gambhir and colleagues in another Article in this issue, fluorescence-guided surgery can help the surgeon navigate through the liver, a feature that is important in deformable tissues because the locations of the lesions to be resected can diverge from those identified via preoperative scans. The researchers describe first-in-human evidence of the performance of a multispectral imaging set-up that combines the detection of fluorescence from the first and second nearinfrared spectral windows $(700-900 \mathrm{~nm}$ and 1,000-1,700 $\mathrm{nm}$ in wavelength, respectively). The imaging system provided higher tumour-detection sensitivity and higher tumour-to-background signal than the sole use of either window, which resulted in higher tumour-detection rates in patients with primary and metastatic liver lesions. Although in practice the system was limited by the use of the non-specific dye indocyanine green for enhanced contrast, it enabled the detection of extrahepatic 
metastases in some patients. These metastatic tumours were not apparent in preoperative scans with enhanced computed tomography, magnetic resonance imaging, ultrasonography and positron emission tomography.
Beyond improved patient outcomes, image-guided surgical systems may also influence the patient's perceived quality of care, which can lead to less anxiety and even reduce the need for second opinions. They may also assist the oncologist to better communicate the potential outcomes, and to comfort and support the patient. That's aid that also counts.

Published online: 12 March 2020

https://doi.org/10.1038/s41551-020-0544-7 